\title{
Novel preparation of substituted oxazolines condensed to D-ring of estrane skeleton and characterization of their antiproliferative properties
}

\author{
Anita Kiss ${ }^{\mathrm{a}}$, Rebeka Jójárt ${ }^{\mathrm{a}}$, Erzsébet Mernyák ${ }^{\mathrm{a}}$, Sándor Bartha ${ }^{\mathrm{b}}$, Renáta Minorics ${ }^{\mathrm{b}}$, \\ István Zupkó $^{\mathrm{b}}$, Gyula Schneider ${ }^{\mathrm{a}, \mathrm{*}}$ \\ ${ }^{a}$ Department of Organic Chemistry, University of Szeged, Dóm tér 8, H-6720 Szeged, Hungary \\ ${ }^{\mathrm{b}}$ Department of Pharmacodynamics and Biopharmacy, University of Szeged, Eötvös $u$. 6, H-6720 Szeged, Hungary
}

\section{A R T I C L E I N F O}

\section{Keywords:}

Heterocyclic steroids

Luche reduction

Schmidt rearrangement

Oxazoline

In vitro inhibition

\begin{abstract}
A B S T R A T
A simple and efficient synthesis of novel estrone $16 \alpha, 17 \alpha$-oxazoline derivatives substituted at the D ring (compounds $6 \mathbf{a - g}$ ) is described. The reduction of $16 \alpha$-azido-3-methoxyestra-1,3,5-trien-17-one (1) in methanol in the presence of $\mathrm{CeCl}_{3}$ under the condition of the Luche reaction produced two epimeric azido alcohol (16 $\alpha$-azido$17 \alpha$-hydroxy and $16 \alpha$-azido-17 $\beta$-hydroxy) derivatives of estra-1,3,5(10)-triene-3-methyl ether (compounds 2 and 3 ) in a yield of $90 \%$ and $7.6 \%$. The reaction of the sterically unhindered $16 \alpha$-azido-17 $\alpha$-hydroxy-estra-1,3,5(10)triene-3-methyl ether (2) with a range of benzaldehydes under the condition of the Schmidt rearrangement yielded D-ring substituted estrone $16 \alpha, 17 \alpha$-oxazoline derivatives 6a-g. The in vitro antiproliferative activities of compounds 1, 2, 3, 6a-g were also determined by means of MTT assays on a panel of human cancer cell lines HeLa, SiHa, C-33 A, A2780, MCF-7, MDA-MB-231 and T47D.
\end{abstract}

\section{Introduction}

Steroids constitute an extensive and important class of biologically active compounds that are widely used for therapeutic purposes [1]. It was found that introducing heterocycles into steroids, modification of the steroidal side chain or substitution of the steroidal skeleton by introducing a heteroatom or replacing one or more carbon atoms in steroidal molecules with a heteroatom, can result in change in biological activities [2]. Steroids containing heteroatoms have been widely researched and reported [3]. Literature reports have suggested that such compounds can display distinct cytotoxicity against cancer cell lines [4].

In view of the potential biological significance of heterocyclic steroids and the well-known antiproliferative potential of numerous steroid derivatives containing oxazoline or dihydrooxazine in different positions on sterane skeleton $[5,6]$, we have decided to prepare arylsubstituted oxazolines condensed to the estrane D-ring in the sterically unhindered $16 \alpha, 17 \alpha$ positions.

Oxazolines represent an attractive group of five-membered heterocyclic compounds as a consequence of their widespread occurrence and diverse pharmacological activities $[7,8]$. As a result of this interest in oxazolines, many useful methods have been developed for the preparation of oxazolines [9].
In our approach to the synthesis of these new type of steroid heterocycles, we used the Schmidt rearrangement using alkyl azides and benzaldehydes in the presence of Lewis acids [10]. In the case of vicinal azidoalcohols, the intermediate of the rearrangement reacts with the sterically favourable neighbouring hydroxy function resulting in a ringclosure reaction to produce an oxazoline moiety condensed to the sterane D-ring in the $16 \alpha, 17 \alpha$ positions.

\section{Experimental}

\subsection{General}

Melting points (Mp) were determined on a Kofler block and are uncorrected. Specific rotations were measured in $\mathrm{CHCl}_{3}$ (c 1) at $20{ }^{\circ} \mathrm{C}$ with a POLAMAT-A (Zeiss-Jena) polarimeter and are given in units of $10^{-1}$ deg $\mathrm{cm}^{2} \mathrm{~g}^{-1}$. Reactions were monitored by thin-layer chromatography (TLC) on Kieselgel-G (Si 254F, Merck KGaA, Darmstadt, Germany) layers ( $0.25 \mathrm{~mm}$ thick); solvent systems (ss): (A) isopropyl ether, (B) acetone/toluene/hexane (30:35:35 v/v). The spots were detected by spraying with $5 \%$ phosphomolybdic acid in $50 \%$ aqueous phosphoric acid. The $R_{\mathrm{f}}$ values were determined for the spots observed by illumination at 254 and $365 \mathrm{~nm}$. Flash chromatography: Merck silica gel 60,

\footnotetext{
* Corresponding author.

E-mail address: schneider@chem.u-szeged.hu (G. Schneider).
} 
40-63 $\mu \mathrm{m}$ (Merck KGaA, Darmstadt, Germany). Elementary analysis data were determined with a PerkinElmer CHN analyser model 2400 (PerkinElmer Inc, Waltham, MA, USA). All solvents were distilled prior to use. Nuclear magnetic resonance (NMR) spectra were recorded on a Bruker DRX 500 and Bruker Ascend 500 instruments at $500\left({ }^{1} \mathrm{H}\right.$ NMR) or $125 \mathrm{MHz}\left({ }^{13} \mathrm{C}\right.$ NMR). Chemical shifts are reported in ppm ( $\delta$ scale), and coupling constants $(J)$ are given in Hertz. For the determination of multiplicities, the $J$-MOD pulse sequence was used.

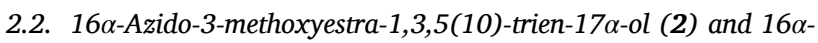 azido-3-methoxyestra-1,3,5(10)-trien-17 $\beta$-ol (3)}

Compound 1 (16 $\alpha$-azido-3-methoxyestra-1,3,5(10)-trien-17-one) [11] (3.25 g, $10 \mathrm{mmol})$ was dissolved in $\mathrm{MeOH}(250 \mathrm{ml})$ containing a few drops of EtOAc. To the stirred solution was added $2.46 \mathrm{~g}$, (10 mmol) anhydrous $\mathrm{CeCl}_{3}$. After $\mathrm{CeCl}_{3}$ was completely dissolved, the solution was cooled to $15{ }^{\circ} \mathrm{C}$. Then $\mathrm{NaBH}_{4}(378 \mathrm{mg}, 10 \mathrm{mmol})$ was added in small portions over $20 \mathrm{~min}$. Vigorous hydrogen gas evolution occurred and a progress of the reaction was monitored by TLC. The mixture, after complete reaction in $20 \mathrm{~min}$, was poured into $5 \%$ aqueous $\mathrm{HCl}$ and extracted with EtOAc $(3 \times 100 \mathrm{ml})$. The extract was washed with saturated aqueous $\mathrm{NaHCO}_{3}$ and water and then dried over $\mathrm{Na}_{2} \mathrm{SO}_{4}$. The solvent was evaporated and the crude product mixture was chromatographed on silica gel column with a mixture of $\mathrm{CH}_{2} \mathrm{Cl}_{2} /$ hexane $(1: 3 \mathrm{v} / \mathrm{v}$ $\%)$ to give pure $2(2.95 \mathrm{~g}, 90 \%)$. Mp.: $96-97{ }^{\circ} \mathrm{C},\left(97-99{ }^{\circ} \mathrm{C}[11]\right), R_{\mathrm{f}}=$ 0.75 (ss B); $[\alpha]_{\mathrm{D}}{ }^{25}=+5$ (c 1 in $\mathrm{CHCl}_{3}$ ). Found: C, 69.56; H, 7.76. $\mathrm{C}_{19} \mathrm{H}_{25} \mathrm{~N}_{3} \mathrm{O}_{2}$ (327.42) requires: $\mathrm{C}, 69.70 ; \mathrm{H}, 7.70 \%{ }^{1} \mathrm{H}$ NMR $(500 \mathrm{MHz}$, $\left.\mathrm{CDCl}_{3}\right): \delta_{\mathrm{H}} 0.76\left(\mathrm{~s}, 3 \mathrm{H}, 18-\mathrm{H}_{3}\right), 2.86\left(\mathrm{~m}, 2 \mathrm{H}, 6-\mathrm{H}_{2}\right), 3.77(\mathrm{~s}, 1 \mathrm{H}, 17-\mathrm{H})$, 3.79 (s, 3H, 3-OMe), 4.20 (dd, $1 \mathrm{H}, J=5.0 \mathrm{~Hz}, J=15.0 \mathrm{~Hz}, 16-\mathrm{H}$ ), 6.63 (d, $1 \mathrm{H}, J=2.0 \mathrm{~Hz}, 4-\mathrm{H}), 6.72$ (dd, $1 \mathrm{H}, J=2.4 \mathrm{~Hz}, J=8.5 \mathrm{~Hz}, 2$ H), 7.21 (d, $1 \mathrm{H}, J=8.5 \mathrm{~Hz}, 1-\mathrm{H}) ;{ }^{13} \mathrm{C}$ NMR $\left(125 \mathrm{MHz}, \mathrm{CDCl}_{3}\right): \delta_{\mathrm{C}} 17.1$ (C-18), 25.7, 28.0, 29.7, 31.0, 31.1, 38.7 (C-8), 43.4 (C-9), 45.6 (C-13), 46.8 (C-14), 55.2 (3-OMe), 63.4 (C-16), 79.3 (C-17), 111.5 (C-2), 113.8 (C-4), 126.2 (C-1), 132.4 (C-10), 137.8 (C-5), 157.5 (C-3). Continued elution with $\mathrm{CH}_{2} \mathrm{Cl}_{2} /$ hexane $(1: 1 \mathrm{v} / \mathrm{v} \%)$ resulted in 3 as a white solid (250 mg, 7.6\%). Mp.: $110-112{ }^{\circ} \mathrm{C}$ (lit.: $112-114{ }^{\circ} \mathrm{C}[11]$ ), $R_{\mathrm{f}}=0.65$ (ss B); $[\alpha]_{D}{ }^{25}=+40\left(c 1\right.$ in $\left.\mathrm{CHCl}_{3}\right)$. Found: C, 69.84; H, 7.58. $\mathrm{C}_{19} \mathrm{H}_{25} \mathrm{~N}_{3} \mathrm{O}_{2}$ (327.42) requires: C, 69.70; H. 7,70\%. ${ }^{1} \mathrm{H}$ NMR (500 MHz, $\left.\mathrm{CDCl}_{3}\right): \delta_{\mathrm{H}}$ $0.83\left(\mathrm{~s}, 3 \mathrm{H}, 18-\mathrm{H}_{3}\right), 2.86\left(\mathrm{~m}, 2 \mathrm{H}, 6-\mathrm{H}_{2}\right), 3.63(\mathrm{~d}, 1 \mathrm{H}, J=6.5 \mathrm{~Hz}, 17-\mathrm{H})$, 3.78 (s, 3H, 3-OMe), 3.82 (m, 1H, 16-H), 6.64 (d, $1 \mathrm{H}, J=2.0 \mathrm{~Hz}, 4-\mathrm{H})$, 6.73 (dd, $1 \mathrm{H}, J=2.0 \mathrm{~Hz}, J=8.5 \mathrm{~Hz}, 2-\mathrm{H}), 7.20$ (d, $1 \mathrm{H}, J=8.5 \mathrm{~Hz}, 1-\mathrm{H}$ ); ${ }^{13} \mathrm{C}$ NMR $\left(125 \mathrm{MHz}, \mathrm{CDCl}_{3}\right): \delta_{\mathrm{C}} 11.9(\mathrm{C}-18), 25.8,27.1,29.6,30.5,36.3$, 38.2, 43.7, 43.8 (C-13), 48.2, 55.2 (3-OMe), 67.0 (C-16), 87.2 (C-17), 111.5 (C-2), 113.8 (C-4), 126.2 (C-1), 132.1 (C-10), 137.7 (C-5), 157.5 (C-3).

2.3. General method for the synthesis of ring D-condensed oxazolines $6 a-$ 9

A solution of compound 2 (1 mmol) and benzaldehyde or 4substituted benzaldehydes (1.1 equivalent) in $\mathrm{CH}_{2} \mathrm{Cl}_{2}(20 \mathrm{ml})$ was cooled to $0{ }^{\circ} \mathrm{C}$, followed by dropwise addition of $\mathrm{BF}_{3} \cdot \mathrm{OEt}_{2}$ (2.0 equivalents). The addition of Lewis acid was accompanied by gas evolution. The reaction mixture was allowed to warm to room temperature and stirred for $2 \mathrm{~h}$. Saturated $\mathrm{NaHCO}_{3}$ solution was added slowly and the mixture was stirred until bubbling ceased. The reaction mixture was extracted with $\mathrm{CH}_{2} \mathrm{Cl}_{2}$, the organic layer was washed with brine, dried over $\mathrm{Na}_{2} \mathrm{SO}_{4}$, concentrated in vacuo to afford the crude product, which was purified by chromatography on silica gel with $\mathrm{CH}_{2} \mathrm{Cl}_{2}$ / hexane $(1: 3$ $\mathrm{v} / \mathrm{v} \%)$.

\subsubsection{3-Methoxy-[(16 $\alpha, 17 \alpha)-2^{\prime}$-phenyl-4', $5^{\prime}$-oxazolinyl]estra-1,3,5(10)- triene $(6 a)$}

Yield $320 \mathrm{mg}(82 \%) . \mathrm{Mp}: 179-181^{\circ} \mathrm{C}, R_{\mathrm{f}}=0.40$ (ss A); $[\alpha]_{\mathrm{D}}{ }^{25}+55(\mathrm{c}$ 1 in $\mathrm{CHCl}_{3}$ ). Found C, 80.34; H, 7.62. $\mathrm{C}_{26} \mathrm{H}_{29} \mathrm{NO}_{2}$ (387.51): requires: C, 80.59; H, 9.54\%. $={ }^{1} \mathrm{H}$ NMR (500 MHz, $\mathrm{CDCl}_{3}$ ): $\delta_{\mathrm{H}} 0.88\left(\mathrm{~s}, 3 \mathrm{H}, 18-\mathrm{H}_{3}\right)$, $2.85\left(\mathrm{~m}, 2 \mathrm{H}, 6-\mathrm{H}_{2}\right), 3.77$ (s, 3H, 3-OCH $3 \mathrm{OMe}$ ), 4.59 (d, $1 \mathrm{H}, J=7.0 \mathrm{~Hz}$, $17-\mathrm{H}), 4.48$ (t, $1 \mathrm{H}, J=7.0 \mathrm{~Hz}, 16-\mathrm{H}), 6.63$ (d, $1 \mathrm{H}, J=1.5 \mathrm{~Hz}, 4-\mathrm{H}),, 6.71$ (dd, $1 \mathrm{H}, J=1.5 \mathrm{~Hz}, J=8.5 \mathrm{~Hz}, 2-\mathrm{H}), 7.21$ (d, $1 \mathrm{H}, J=8.5 \mathrm{~Hz}, 1-\mathrm{H}), 7.43$ (t, $2 \mathrm{H}, J=7.0 \mathrm{~Hz}, 3^{\prime \prime}$ - and $\left.5^{\prime \prime}-\mathrm{H}\right), 7.49$ (t, $1 \mathrm{H}, J=7.0 \mathrm{~Hz}, 4^{\prime \prime}-\mathrm{H}$ ), 7.99 (d, $2 \mathrm{H}, J=7.0 \mathrm{~Hz}, 2^{\prime \prime}$ - and $\left.6^{\prime \prime}-\mathrm{H}\right) .={ }^{13} \mathrm{C}$ NMR $\left(125 \mathrm{MHz}, \mathrm{CDCl}_{3}\right): \delta_{\mathrm{C}} 17.6(\mathrm{C}-$ 18), 26.0, 28.0, 29.8, 32.6, 34.0, 38.2, 43.4, 45.5 (C-13), 46.4, 55.2 (3OMe), 70.1 (C-16), 91.2 (C-17), 111.5 (C-2), 113.7 (C-4), 126.2 (C-1), $127.7\left(\mathrm{C}-1^{\prime \prime}\right), 128.3\left(\mathrm{C}-2^{\prime \prime}\right.$ and $\left.-6^{\prime \prime}\right), 128.3\left(\mathrm{C}-3^{\prime \prime}\right.$ and $\left.-5^{\prime \prime}\right), 131.3\left(\mathrm{C}-4^{\prime \prime}\right)$, 132.4 (C-10), 137.9 (C-5), 157.4 (C-3), 164.1 (C-2').

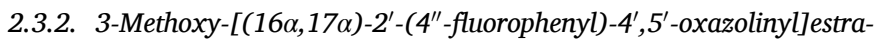 $1,3,5(10)$-triene $(6 b)$}

Yield $310 \mathrm{mg}(76 \%)$. Mp: $171-173{ }^{\circ} \mathrm{C}, R_{\mathrm{f}}=0.45$ (ss A); $[\alpha]_{\mathrm{D}}{ }^{25}+42$ (c 1 in $\mathrm{CHCl}_{3}$ ). Found: $\mathrm{C}, 76.92 ; \mathrm{H}, 6.38 . \mathrm{C}_{26} \mathrm{H}_{28} \mathrm{FNO}_{2}$ (405.50) requires: C, 77.01; H, 6.96\%. $={ }^{1} \mathrm{H}$ NMR (500 MHz, $\left.\mathrm{CDCl}_{3}\right): \delta_{\mathrm{H}} 0.88$ (s, 3H, 18$\mathrm{H}_{3}$ ), $2.83\left(\mathrm{~m}, 2 \mathrm{H}, 6-\mathrm{H}_{2}\right), 3.77$ (s, 3H, 3-OMe), 4.59 (d, $1 \mathrm{H}, J=7.0 \mathrm{~Hz}, 17-$ $\mathrm{H}), 4.76(\mathrm{t}, 1 \mathrm{H}, J=7.0 \mathrm{~Hz}, 16-\mathrm{H}), 6.62(\mathrm{~d}, 1 \mathrm{H}, J=2.5 \mathrm{~Hz}, 4-\mathrm{H}), 6.71$ (dd, $1 \mathrm{H},, J=2.5 \mathrm{~Hz}, J=8.5 \mathrm{~Hz}, 2-\mathrm{H}), 7.10$ (t, $2 \mathrm{H}, J=9.0 \mathrm{~Hz}, 3^{\prime \prime}$ - and $5^{\prime \prime}-\mathrm{H}$ ), 7.20 (d, $1 \mathrm{H}, J=8.5 \mathrm{~Hz}, 1-\mathrm{H}), 7.97$ (dd, $1 \mathrm{H}, J=9.0 \mathrm{~Hz}, J=5.5 \mathrm{~Hz}, J=$ $5.5 \mathrm{~Hz}, J=9.0 \mathrm{~Hz}, 2^{\prime \prime}$ - and $\left.6^{\prime \prime}-\mathrm{H}\right) .{ }^{13} \mathrm{C}$ NMR $\left(125 \mathrm{MHz}, \mathrm{CDCl}_{3}\right): \delta_{\mathrm{C}} 17.6$ (C-18), 25.9, 28.0, 29.8, 32.6, 34.0, 38.2, 43.4, 45.5 (C-13), 46.4, 55.2 (3-OMe), 70.3 (C-16), 91.4 (C-17), 111.5 (C-2), 113.7 (C-4), 115.4 (d, 2C, $J=21.9 \mathrm{~Hz}, \mathrm{C}-3^{\prime \prime}$ and C-5"), 124.1 (C-1"), 126.3 (C-1), 130.4 (d, 2C, $J=8.8 \mathrm{~Hz}, \mathrm{C}-2^{\prime \prime}$ and C-6"), 132.4 (C-10), 137.9 (C-5), 157.5 (C-3), 163.1 (C-2'), 164.6 (d, $J=251.6 \mathrm{~Hz}, \mathrm{C}-4^{\prime \prime}$ ).

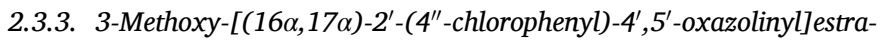 $1,3,5(10)$-triene $(6 c)$}

Yield $355 \mathrm{mg}$ (84\%). Mp: $174-176^{\circ} \mathrm{C}, R_{\mathrm{f}}=0.45$ (ss A); $[\alpha]_{\mathrm{D}}{ }^{25}+44$ (c 1 in $\mathrm{CHCl}_{3}$ ). Found $\mathrm{C}, 74.16 ; \mathrm{H}, 6.82 . \mathrm{C}_{26} \mathrm{H}_{28} \mathrm{ClNO}_{2}$ (421.96) requires: C, 74.01; H, 6.69\%. $={ }^{1} \mathrm{H}$ NMR (500 MHz, $\mathrm{CDCl}_{3}$ ): $\delta_{\mathrm{H}} 0.88$ (s, 3H, 18$\mathrm{H}_{3}$ ), 2.85 (m, $2 \mathrm{H}, 6-\mathrm{H}_{2}$ ), 3.77 (s, 3H, 3-OMe), 4.59 (d, $1 \mathrm{H}, J=7.5 \mathrm{~Hz}, 17-$ H), 4.77 (t, $1 \mathrm{H}, J=7.5 \mathrm{~Hz}, 16-\mathrm{H}), 6.62(\mathrm{~d}, 1 \mathrm{H}, J=2.5 \mathrm{~Hz}, 4-\mathrm{H}), 6.71$ (dd, $1 \mathrm{H},, J=2.5 \mathrm{~Hz}, J=8.5 \mathrm{~Hz}, 2-\mathrm{H}$ ), 7.20 (d, $1 \mathrm{H}, J=8.5 \mathrm{~Hz}, 1-\mathrm{H}$ ), 7.40 (d, $2-\mathrm{H}), J=8.5 \mathrm{~Hz}, 3^{\prime \prime}$ - and $\left.5^{\prime \prime}-\mathrm{H}\right), 7.91$ (d, $2 \mathrm{H}, J=8.5 \mathrm{~Hz}, 2^{\prime \prime}$ - and $\left.6^{\prime \prime}-\mathrm{H}\right)$. ${ }^{13} \mathrm{C}$ NMR (125 MHz, $\mathrm{CDCl}_{3}$ ): $\delta_{\mathrm{C}} 17.6$ (C-18), 25.9, 28.0, 29.8, 32.6, 34.0, 38.2, 43.4, 45.5 (C-13), 46.4, 55.2 (3-OMe), 70.2 (C-16), 91.4 (C-17), 111.5 (C-2), 113.7 (C-4), 126.1 (C-1"), 126.2 (C-1), 128.6 (C-2" and $\left.-6^{\prime \prime}\right), 129.6\left(\mathrm{C}-3^{\prime \prime}\right.$ and $\left.-5^{\prime \prime}\right), 132.3(\mathrm{C}-10), 137.5\left(\mathrm{C}-4^{\prime \prime}\right), 137.9(\mathrm{C}-5)$, 157.5 (C-3), $163.3\left(\mathrm{C}-2^{\prime}\right)$.

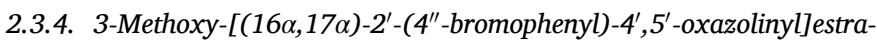 1,3,5(10)-triene (6d)}

Yield $370 \mathrm{mg}$ (79\%). Mp: $258-260{ }^{\circ} \mathrm{C}, R_{\mathrm{f}}=0.43$ (ss A); $[\alpha]_{\mathrm{D}}{ }^{25}+48$ (c 1 in $\mathrm{CHCl}_{3}$ ). Found: $\mathrm{C}, 67.12 ; \mathrm{H}, 6.32 . \mathrm{C}_{26} \mathrm{H}_{28} \mathrm{BrNO}_{2}$ (466.41) requires: $\mathrm{C}, 66.95 ; \mathrm{H}, 6.05 \% .={ }^{1} \mathrm{H} \mathrm{NMR}\left(500 \mathrm{MHz}, \mathrm{CDCl}_{3}\right): \delta_{\mathrm{H}} 0.88(\mathrm{~s}, 3 \mathrm{H}$, 18- $\mathrm{H}_{3}$ ), 2.85 (m, 2H, 6- $\mathrm{H}_{2}$ ), 3.77 (s, 3H, 3-OMe), 4.60 (d, $1 \mathrm{H}, J=7.0 \mathrm{~Hz}$, 17-H), 4.77 (t, $1 \mathrm{H}, J=7.0 \mathrm{~Hz}, 16-\mathrm{H}), 6.62$ (s, 1H, 4-H), 6.71 (d, $1 \mathrm{H}, J=$ $8.5 \mathrm{~Hz}, 2-\mathrm{H}), 7.20$ (d, $1 \mathrm{H}, J=8.5 \mathrm{~Hz}, 1-\mathrm{H}), 7.56$ (d, $2 \mathrm{H}, J=8.5 \mathrm{~Hz}, 3^{\prime \prime}$ and $\left.5^{\prime \prime}-\mathrm{H}\right), 7.85$ (d, $2 \mathrm{H}, J=8.5 \mathrm{~Hz}, 2^{\prime \prime}-$ and $\left.6^{\prime \prime}-\mathrm{H}\right) .{ }^{13} \mathrm{C}$ NMR $(125 \mathrm{MHz}$, $\left.\mathrm{CDCl}_{3}\right): \delta_{\mathrm{C}} 17.6(\mathrm{C}-18), 25.9,28.0,29.8,32.6,34.0,38.2,43.4,45.6(\mathrm{C}-$ 13), 46.5, 55.2 (3- OMe), 70.1 (C-16), 91.5 (C-17), 111.5 (C-2), 113.7 (C4), $126.1\left(\mathrm{C}-1^{\prime \prime}\right), 126.2(\mathrm{C}-1), 126.4\left(\mathrm{C}-4^{\prime \prime}\right), 129.9\left(\mathrm{C}-2^{\prime \prime}\right.$ and $\left.-6^{\prime \prime}\right), 131.6$ (C-3" and -5"), 132.3 (C-10), 137.9 (C-5), 157.5 (C-3), $163.5\left(\mathrm{C}-2^{\prime}\right)$.

\subsubsection{3-Methoxy-[(16, $17 \alpha)-2^{\prime}-\left(4^{\prime \prime}-\right.$ methoxyphenyl)-4',5'-oxazolinyl] estra-1,3,5(10)-triene (6e)}

Yield $293 \mathrm{mg}$ (70\%). Mp: $169-170{ }^{\circ} \mathrm{C}, R_{\mathrm{f}}=0.35$ (ss A); $[\alpha]_{\mathrm{D}}{ }^{25}+51$ (c 1 in $\mathrm{CHCl}_{3}$ ). Found: $\mathrm{C}, 77.82 ; \mathrm{H}, 7.36 . \mathrm{C}_{27} \mathrm{H}_{31} \mathrm{NO}_{3}$ (417.54) requires: C, 77.67; H, 7.48\%. ${ }^{1} \mathrm{H}$ NMR (500 MHz, $\mathrm{CDCl}_{3}$ ): $\delta_{\mathrm{H}} 0.87$ (s, 3H, $18-\mathrm{H}_{3}$ ), 2.85 (m, 2H, 6- $\mathrm{H}_{2}$ ), 3.77 (s, 3H, 3-OMe), 3.85 (s, 3H, 4"-OMe), 4.56 (d, $1 \mathrm{H}, J=7.0 \mathrm{~Hz}, 17-\mathrm{H}), 4.75(\mathrm{t}, 1 \mathrm{H}, J=7.0 \mathrm{~Hz}, 16-\mathrm{H}), 6.63$ (d, $1 \mathrm{H}, J=1.5$ $\mathrm{Hz}, 4-\mathrm{H}), 6.71$ (dd, $1 \mathrm{H}, J=8.5 \mathrm{~Hz}, 2-\mathrm{H}), 6.93$ (d, $2 \mathrm{H}, J=9.0 \mathrm{~Hz}, 3^{\prime \prime}$ - and $\left.5^{\prime \prime}-\mathrm{H}\right), 7.20(\mathrm{~d}, 1 \mathrm{H}, J=8.5 \mathrm{~Hz}, 1-\mathrm{H}), 7.93$ (d, $2 \mathrm{H}, J=9.0 \mathrm{~Hz}, 2^{\prime \prime}$ - and $6^{\prime \prime}$ H). ${ }^{13} \mathrm{C}$ NMR (125 MHz, $\mathrm{CDCl}_{3}$ ): $\delta_{\mathrm{C}} 17.6$ (C-18), 26.0, 28.0, 29.8, 32.6, 34.1, 38.2, 43.4, 45.5 (C-13), 46.3, 55.1 (3-OMe), 55.3 (4"-OMe), 70.0 
<smiles>COc1ccc2c(c1)CC[C@@H]1[C@@H]2CC[C@]2(C)C(=O)[C@H](N)C[C@H]12</smiles><smiles>COc1ccc2c(c1)CC[C@H]1[C@@H]2CC[C@]2(C)[C@H](O)[C@@H](N)C[C@H]12</smiles>

\begin{tabular}{|c|c|c|c|}
\hline \multicolumn{4}{|c|}{$\begin{array}{l}\text { Reduction of } 1 \text { under different } \\
\text { conditions }\end{array}$} \\
\hline \multirow[t]{2}{*}{ Entry } & \multicolumn{2}{|c|}{ Compounds (\%) } & Ref \\
\hline & 2 & 3 & \\
\hline 1 & 67 & 13 & [11] \\
\hline 2 & 42 & 54 & [16] \\
\hline 3 & 90 & 7.6 & [Present] \\
\hline
\end{tabular}

Scheme 1. Reagents and conditions: (i): [11]: $\mathrm{Et}_{2} \mathrm{O} ; \mathrm{KBH}_{4} ;[16]: \mathrm{MeOH} / \mathrm{CH}_{2} \mathrm{Cl}_{2}$ (v/v); $\mathrm{NaBH}_{4} ;$ [Present]: $\mathrm{MeOH} ; \mathrm{CeCl}_{3} ; \mathrm{NaBH}_{4}$.

(C-16), 91.0 (C-17), 111.5 (C-2), 113.7 (C-4), 113.7 (C-2" és and -6"), $120.2\left(\mathrm{C}-1^{\prime \prime}\right), 126.2(\mathrm{C}-1), 130.0\left(\mathrm{C}-3^{\prime \prime}\right.$ and $\left.-5^{\prime \prime}\right), 132.5(\mathrm{C}-10), 137.9$ (C5), 157.4 (C-3), 162.0 (C-2'), 163.8 (C-4").

\subsubsection{3-Methoxy-[(16 $\alpha, 17 \alpha)-2^{\prime}-\left(4^{\prime \prime}\right.$-cyanophenyl)-4',5'-oxazolinyl]estra- $1,3,5(10)$-triene (6f)}

Yield $258 \mathrm{mg}(62 \%) . \mathrm{Mp}: 208-210^{\circ} \mathrm{C}, R_{\mathrm{f}}=0.38(\mathrm{ss} \mathrm{A}) ;[\alpha]_{\mathrm{D}}{ }^{20}+48(\mathrm{c}$ 1 in $\mathrm{CHCl}_{3}$ ). Found: $\mathrm{C}, 78.70 ; \mathrm{H}, 6.92 . \mathrm{C}_{27} \mathrm{H}_{28} \mathrm{~N}_{2} \mathrm{O}_{2}$ (412.52) requires: $\mathrm{C}$, 78.61; H, 6.84\%. ${ }^{1} \mathrm{H}$ NMR (500 MHz, $\mathrm{CDCl}_{3}$ ): $\delta_{\mathrm{H}} 0.89\left(\mathrm{~s}, 3 \mathrm{H}, 18-\mathrm{H}_{3}\right.$ ), $2.84\left(\mathrm{~m}, 2 \mathrm{H}, 6-\mathrm{H}_{2}\right), 3.77$ (s, 3H, 3-OMe), $4.62(\mathrm{~d}, 1 \mathrm{H}, J=7.0 \mathrm{~Hz}, 17-\mathrm{H})$, 4.81 (t, $1 \mathrm{H}, J=7.0 \mathrm{~Hz}, 16-\mathrm{H}), 6.62$ (s, $1 \mathrm{H}, 4-\mathrm{H}), 6.71$ (dd, $1 \mathrm{H}, J=1.5 \mathrm{~Hz}$, $J=1.5 \mathrm{~Hz}, J=8.5 \mathrm{~Hz}, 2-\mathrm{H}), 7.20$ (d, $1 \mathrm{H}, J=8.5 \mathrm{~Hz}, 1-\mathrm{H}), 7.71$ (d, $2 \mathrm{H}, J$ $=8.5 \mathrm{~Hz}, 3^{\prime \prime}$ - and $\left.5^{\prime \prime}-\mathrm{H}\right), 8.08\left(\mathrm{~d}, 1 \mathrm{H} 2 \mathrm{H}, J=8.5 \mathrm{~Hz}, 2^{\prime \prime}\right.$ - and $\left.6^{\prime \prime}-\mathrm{H}\right) .{ }^{13} \mathrm{C}$ NMR (125 MHz, $\left.\mathrm{CDCl}_{3}\right): \delta_{\mathrm{C}} 17.5$ (C-18), 25.9, 28.0, 29.7, 32.5, 33.9, 38.1, 43.4, 45.6 (C-13), 46.5, 55.2 (3-OMe), 70.4 (C-16), 91.7 (C-17), 111.5 (C-2), 113.7 (C-4), 114.6 (C-4"), 118.3 (-CN), 126.2 (C-1), 128.8 $\left(\mathrm{C}-2^{\prime \prime}\right.$ and $\left.-6^{\prime \prime}\right), 131.8\left(\mathrm{C}-1^{\prime \prime}\right), 132.1\left(\mathrm{C}-3^{\prime \prime}\right.$ and $\left.-5^{\prime \prime}\right), 132.2$ (C-10), 137.8 (C-5), 157.5 (C-3), 162.5 (C-2').

\subsubsection{3-Methoxy-[(16 $\alpha, 17 \alpha-2^{\prime}-\left(4^{\prime \prime}\right.$-lnitrophenyl)-4',5'-oxazolinyl]estra-} 1,3,5(10)-triene $(6 \mathrm{~g})$

Yield $248 \mathrm{mg}(57 \%)$. Mp: $217-219{ }^{\circ} \mathrm{C}, R_{\mathrm{f}}=0.32$ (ss A); $[\alpha]_{\mathrm{D}}{ }^{25}+49$ (c 1 in $\mathrm{CHCl}_{3}$ ). Found: $\mathrm{C}, 72.05 ; \mathrm{H}, 6.62 . \mathrm{C}_{26} \mathrm{H}_{28} \mathrm{~N}_{2} \mathrm{O}_{4}$ (432.51) requires: C, 72.20; H, 6.53\%. ${ }^{1} \mathrm{H}$ NMR (500 MHz, $\mathrm{CDCl}_{3}$ ): $\delta_{\mathrm{H}} 0.90$ (s, 3H, 18- $\mathrm{H}_{3}$ ), $2.86\left(\mathrm{~m}, 2 \mathrm{H}, 6-\mathrm{H}_{2}\right), 3.77$ (s, 3H, 3-OMe), 4.65 (d, $\left.1 \mathrm{H}, J=7.0 \mathrm{~Hz}, 17-\mathrm{H}\right)$, 4.83 (t, $1 \mathrm{H}, J=7.0 \mathrm{~Hz}, 16-\mathrm{H}), 6.62$ (d, $1 \mathrm{H}, J=2.5 \mathrm{~Hz}, 4-\mathrm{H}), 6.71$ (dd, $1 \mathrm{H}, J=8.5 \mathrm{~Hz}, J=2.5 \mathrm{~Hz}, 2-\mathrm{H}), 7.20$ (d, $1 \mathrm{H}, J=8.5 \mathrm{~Hz}, 1-\mathrm{H}), 8.15$ (d, $2 \mathrm{H}, J=8.5 \mathrm{~Hz}, 3^{\prime \prime}$ - and $\left.5^{\prime \prime}-\mathrm{H}\right), 8.27\left(\mathrm{~d}, 2 \mathrm{H}, J=8.5 \mathrm{~Hz}, 2^{\prime \prime}-\right.$ and $\left.6^{\prime \prime}-\mathrm{H}\right) .{ }^{13} \mathrm{C}$ NMR (125 MHz, $\mathrm{CDCl}_{3}$ ): $\delta_{\mathrm{C}} 17.6$ (C-18), 25.9, 28.0, 29.7, 32.6, 33.9, 38.1, 43.4, 45.6 (C-13), 46.5, 55.2 (3-OMe), 70.5 (C-16), 91.9 (C-17), 111.5 (C-2), 113.7 (C-4), 123.5 (C-2" and -6"), 126.2 (C-1), 129.3 (C-3" and $\left.-5^{\prime \prime}\right), 132.2(\mathrm{C}-10), 133.5\left(\mathrm{C}-1^{\prime \prime}\right), 137.8(\mathrm{C}-5), 149.4\left(\mathrm{C}-4^{\prime \prime}\right), 157.5$ (C3), $162.4\left(\mathrm{C}-2^{\prime}\right)$.

\subsection{Antiproliferative MTT assay}

For the antiproliferative screening, a panel of human adherent gynecological cancer cell lines was used. The human breast cancer cell lines (MCF-7, MDA-MB-231 and T47D), ovarian carcinoma (A2780) and HPV 18 + cervical adenocarcinoma (HeLa) cell lines were purchased from ECACC (European Collection of Cell Cultures, Salisbury, UK), while SiHa (HPV 16 + squamous cell carcinoma) and C33 A (HPV - epithelial carcinoma) were purchased from ATCC (American Tissue Culture Collection, LGC Standards GmbH, Wesel, Germany). Cells were maintained in Eagle's Minimum Essential Medium (EMEM) supplemented with $10 \%$ heat-inactivated fetal calf serum (FCS), $1 \%$ nonessential amino acids (NEAA), and $1 \%$ antibiotic-antimycotic mixture (AAM, penicillin-streptomycin). All media and supplements were obtained from Lonza Group Ltd. (Basel, Switzerland). The cells were maintained at $37^{\circ} \mathrm{C}$ in humidified atmosphere containing $5 \% \mathrm{CO}_{2}$.

The growth-inhibitory effect of the compounds was determined by standard MTT (3-(4,5-dimethylthiazol-2-yl)-2,5-diphenyltetrazolium bromide) dye uptake method. Briefly, cells were seeded onto 96well plates at a density of 5000 cells/well, except for C33 A which were seeded at 10,000/cells/well. After overnight standing, new medium, containing the tested compounds in concentrations of 10 and $30 \mu \mathrm{M}$, was added for the antiproliferative screening followed by adding increasing concentrations of the test compounds $(0.130 .0 \mu \mathrm{M})$ to determine concentration-response curves. After incubation for $72 \mathrm{~h}$ under cell culturing conditions, $5 \mathrm{mg} / \mathrm{ml}$ MTT solution was added followed by treatment for another $4 \mathrm{~h}$. The precipitated formazan crystals were solubilised in dimethyl sulfoxide and the absorbance was measured at $545 \mathrm{~nm}$ with a microplate reader (SPECTROstar Nano, BMG Labtech $\mathrm{GmbH}$, Offenburg, Germany). Wells with untreated cells were utilised as control [12] and cisplatin was used as a positive control in the same concentration range as that of the test compounds. Sigmoidal concentration-response curves were fitted to the determined points, and $\mathrm{IC}_{50}$ values were calculated by means of GraphPad Prism 5.01 (GraphPad Software, San Diego, CA, USA).

\section{Results and discussion}

\subsection{Synthetic studies}

To prepare novel steroids with condensed oxazoline ring at position $16 \alpha, 17 \alpha$ of the estrane skeleton, we chose cyclisation of the $16 \alpha, 17 \alpha$ azidoalcohol 2 with, benzaldehyde, and or different 4-substituted benzaldehydes, in the presence of $\mathrm{BF}_{3} \cdot \mathrm{OEt}_{2}$. The synthetic strategy for the preparation of the starting azidoalkohols and the cyclisation process is illustrated in Scheme 1 and Scheme 2.

The reaction between a carbonyl compound (ketone or aldehyde) and hydrazoic acid is a useful method for the insertion of an $\mathrm{NH}$ group between the carbonyl group and an alkyl group, converting the starting compound into a carboxamide [13]. In an extension of this classic Schmidt reaction, Boyer and Hammer found that the reactions of alkyl azides with aromatic aldehydes could be carried out with Brønsted acids to give amides in moderate yields. In contrast, the use of 1,2- or 1,3-azidoalcohols under similar conditions afforded oxazolines or 


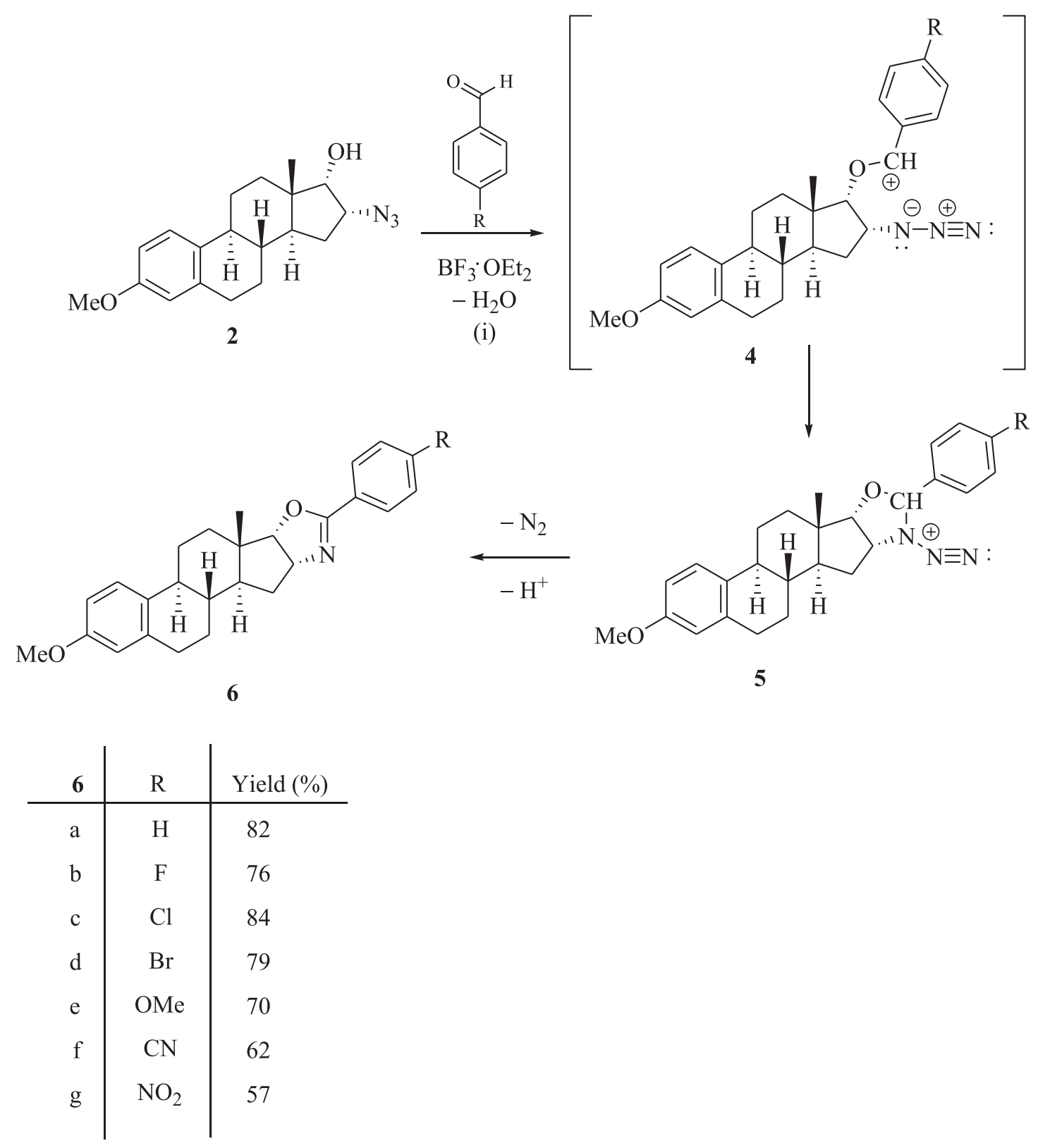

Scheme 2. Reagents and conditions: (i); $\mathrm{BF}_{3} \cdot \mathrm{OEt}_{2} ; \mathrm{CH}_{2} \mathrm{Cl}_{2} ;$ rt.

dihydrooxazines, respectively, with much greater efficiency [14]. Later, utilisation of a variety of Lewis acids was examined, of which $\mathrm{BF}_{3} . \mathrm{OEt}_{2}$ was found to be most convenient [15]. This observation provides a possibility for preparation of compounds containing various substituted oxazolines condensed to D-ring of the estrane skeleton.

To prepare $16 \alpha$-azido-3-methoxyestra-1,3,5(10)-trien-17 $\alpha$-ol (2), we selected 16 $\alpha$-azido-3-methoxyestra-1,3,5(10)-trien-17-one (1) as starting material [11]. The reduction of $16 \alpha$-azido-17-ketone 1 depends strongly on the reduction conditions. Schönecker and Ponsold [11] found that the reduction of $\mathbf{1}$ in diethyl ether with $\mathrm{KBH}_{4}$ affords a mixture of $16 \alpha$-azido-3-methoxyestra-1,3,5(10)-trien-17 $\alpha$-ol (2) and $16 \alpha$-azido-3-methoxyestra-1,3,5(10)-trien-17 $\beta$-ol (3) in a yield of $67 \%$ and $13 \%$, respectively. Frank and al. [16] changed diethyl ether to a mixture of $\mathrm{MeOH} / \mathrm{CH}_{2} \mathrm{Cl}_{2}(4: 1 \mathrm{v} / \mathrm{v})$ and these conditions, using $\mathrm{KBH}_{4}$ yielded $42 \%$ of 2 and $54 \%$ of 3 [16]. In all cases, pure epimers were separated by flash chromatography.

Because the ring closure reaction required the cis position of the $16 \alpha-$ azido, $17 \alpha$-hydroxy functional groups, we wanted to change the ratio of pseudo axial and pseudo equatorial 17 hydroxyl function during the
$\mathrm{NaBH}_{4}$ reduction. Luche published an efficient method for the regioselective reduction of $\alpha, \beta$-unsaturated ketones based on treatment of an equimolar amount of ketone and lanthanoid chloride in $\mathrm{MeOH}$ with $\mathrm{NaBH}_{4}$. Lanthanoid addition allows a highly regioselective 1,2-reduction competing with the undesirable 1,4-reduction [17]. Only a few examples can be found in the literature that use this method for the reduction of saturated ketone to promote the formation of an equatorial over an axial alcohol. This effect is most important in the case of sterically crowded ketones [18]. In 2010, a series of keto steroids were reduced with $\mathrm{NaBH}_{4}$ in the presence of lanthanoid chlorides in $\mathrm{MeOH}$ by Chodounská et al [19]. They found, that the $\mathrm{NaBH}_{4}$ reduction of some ketosteroids under the conditions of the Luche reduction resulted in the inversion of the axial/equatorial ratios [19].

In our case, an equimolar amount of anhydrous $\mathrm{CeCl}_{3}$ was added to the $\mathrm{MeOH}$ solution of the $16 \alpha$-azido-3-methoxyestra-1,3,5(10)-trien-17one (1). The mixture was allowed to stir at room temperature until all $\mathrm{CeCl}_{3}$ dissolved followed by adding an equimolar amount of $\mathrm{NaBH}_{4}$ in small portions. Vigorous hydrogen gas evolution occurred and the reduction was completed in $20 \mathrm{~min}$. The attack by a cerium ion on the 
Table 1

Antiproliferative properties of compounds 1, 2 and 6a-g.

\begin{tabular}{|c|c|c|c|c|c|c|c|c|}
\hline \multirow[t]{2}{*}{ Comp. } & \multirow[t]{2}{*}{ Conc. $(\mu \mathrm{M})$} & \multicolumn{7}{|c|}{ Growth inhibition; $\% \pm$ SEM [calculated $\mathrm{IC}_{50}$ value; $\mu \mathrm{M}$ ] } \\
\hline & & HeLa & $\mathrm{SiHa}$ & C-33 A & A2780 & MCF-7 & MDA-MB-231 & T47D \\
\hline \multirow[t]{2}{*}{1} & 10 & $31.46 \pm 2.8$ & & $33.5 \pm 1.8$ & $26.1 \pm 1.6$ & $23.2 \pm 2.1$ & $34.7 \pm 1.9$ & $36.4 \pm 2.4$ \\
\hline & 30 & & & & & & & \\
\hline \multirow[t]{2}{*}{2} & 10 & $36.8 \pm 3.1$ & & $41.2 \pm 1.8$ & $34.4 \pm 2.8$ & & $33.6 \pm 2.7$ & $40.8 \pm 1.2$ \\
\hline & 30 & & & & & & & \\
\hline \multirow[t]{2}{*}{$6 \mathbf{a}$} & 10 & $31.5 \pm 1.6$ & $34.4 \pm 1.1$ & $20.6 \pm 1.9$ & & $20.2 \pm 1.3$ & $39.8 \pm 2.4$ & $21.6 \pm 2.5$ \\
\hline & 30 & $48.0 \pm 1.6$ & & & & $45.0 \pm 2.0$ & & $48.3 \pm 2.7$ \\
\hline \multirow[t]{2}{*}{$6 b$} & 10 & $55.8 \pm 0.5$ & $23.9 \pm 2.1$ & $61.7 \pm 2.7$ & $31.7 \pm 2.3$ & $26.6 \pm 3.0$ & $30.9 \pm 1.9$ & $34.0 \pm 2.3$ \\
\hline & 30 & & & & & & & \\
\hline \multirow[t]{2}{*}{$6 c$} & 10 & $37.1 \pm 2.9$ & $36.1 \pm 0.6$ & $23.1 \pm 1.1$ & $32.5 \pm 2.3$ & $27.3 \pm 2.9$ & $38.8 \pm 1.1$ & $43.4 \pm 2.3$ \\
\hline & 30 & $62.3 \pm 1.4$ & $54.9 \pm 0.9$ & & & $50.6 \pm 3.1$ & $67.3 \pm 1.8$ & \\
\hline \multirow[t]{2}{*}{$6 d$} & 10 & $49.5 \pm 0.9$ & $28.1 \pm 1.5$ & $42.3 \pm 2.6$ & & $49.4 \pm 0.5$ & $36.2 \pm 1.4$ & $49.7 \pm 0.4$ \\
\hline & 30 & & & & & & & \\
\hline \multirow[t]{2}{*}{$6 e$} & 10 & $35.0 \pm 2.4$ & $48.8 \pm 1.8$ & $24.3 \pm 2.2$ & $28.6 \pm 1.4$ & $51.3 \pm 2.0$ & $38.9 \pm 1.2$ & $49.1 \pm 2.6$ \\
\hline & 30 & $\begin{array}{l}76.7 \pm 1.1 \\
{[12.97]}\end{array}$ & $67.9 \pm 1.1$ & $50.34 \pm 1.4$ & $43.5 \pm 3.1$ & $71.0 \pm 1.3$ & $68.7 \pm 1.1$ & $73.7 \pm 1.3$ \\
\hline \multirow[t]{2}{*}{$6 f$} & 10 & $43.6 \pm 3.1$ & $29.7 \pm 0.6$ & $39.65 \pm 2.2$ & $33.9 \pm 3.1$ & $23.6 \pm 1.03$ & $35.0 \pm 1.9$ & $28.2 \pm 2.4$ \\
\hline & 30 & & $53.0 \pm 1.1$ & & & $51.7 \pm 1.4$ & $60.6 \pm 2.3$ & $45.2 \pm 1.2$ \\
\hline \multirow[t]{2}{*}{$6 g$} & 10 & $32.8 \pm 1.4$ & $43.0 \pm 2.1$ & $28.8 \pm 2.9$ & $34.5 \pm 2.6$ & $42.8 \pm 2.0$ & $22.3 \pm 1.2$ & $39.0 \pm 1.7$ \\
\hline & 30 & $52.0 \pm 2.6$ & $72.61 \pm 1.79$ & $46.6 \pm 2.5$ & $65.9 \pm 2.2$ & $\begin{array}{l}80.7 \pm 1.4 \\
{[16.5]}\end{array}$ & $35.5 \pm 1.9$ & $72.6 \pm 1.9$ \\
\hline \multirow[t]{2}{*}{ CIS } & 10 & $42.6 \pm 2.3$ & $88.6 \pm 0.5$ & $85.9 \pm 1.0$ & 83. \pm 1 & $66.9 \pm 1.8$ & $71.4 \pm 1.2$ & $51.0 \pm 2.0$ \\
\hline & 30 & $\begin{array}{l}99.9 \pm 0.2 \\
{[12.4]}\end{array}$ & $\begin{array}{l}90.18 \pm 1.7 \\
{[7.8]}\end{array}$ & $\begin{array}{l}98.6 \pm 0.2 \\
{[4.1]}\end{array}$ & $\begin{array}{l}95.0 \pm 0.3 \\
{[1.3]}\end{array}$ & $\begin{array}{l}96.8 \pm 0.3 \\
{[5.7]}\end{array}$ & [19.1] & $\begin{array}{l}57.9 \pm 1.4 \\
{[9.7]}\end{array}$ \\
\hline
\end{tabular}

*These growth inhibition values are less than $20 \%$ which was considered as negligible.

${ }^{\#} \mathrm{IC}_{50}$ values have been calculated if the growth inhibition value of the compound at $30 \mu \mathrm{M}$ concentration is higher than $75 \%$.

carbonyl oxygen allows the formation of a cerium-steroid complex that distinctively enhances the attack of the borohydride anion and the subsequent formation of a $17 \alpha$-hydroxyl function. Furthermore the presence of $16 \alpha$-azido group with steric hindrance promotes also the attack of borohydride anion by the steroid $\beta$ - face. The vigorous gas evolution indicates the formation of alkoxyborohydrides, the actual reducing species. In the case of Luche reduction of $\mathbf{1}$, the product ratio was completely reversed when compared to that of the standard borohydride reduction. Specifically, the addition of $\mathrm{CeCl}_{3}$ into the reaction mixture increased the amount of the required $17 \alpha$ epimer 2 from $42 \%$ to $90 \%$, with the concomitant decrease of the amount of the $17 \beta$ epimer 3 from $54 \%$ to $7.6 \%$.

We reported earlier, that the acid-catalysed reactions of $3 \beta$-acetoxy21 -azidopregn-5-en-20 $\beta$-ol, and $3 \beta$-acetoxy-20 $\alpha$-azidopregn-5-en-21-ol with substituted aromatic aldehydes under the condition of Schmidt

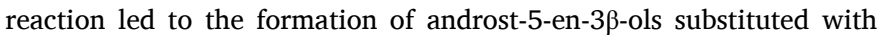
oxazoline moieties in the $17 \beta$ position [5]. On the basis of this earlier observation, we set out to synthesize a novel series of substituted oxazolines condensed to the estrane skeleton. This method allowed the transformation of the $16 \alpha, 17 \alpha$-azidolakohol 2 under the Schmidt reaction conditions to deliver $16 \alpha, 17 \alpha$-condensed oxazolines 6a-g.

Starting from the mixture of $16 \alpha$-azido-3-methoxyestra-1,3,5(10)trien-17 $\alpha$-ol (2) having the suitable stereochemical arrangement and the corresponding substituted benzaldehyde in $\mathrm{CH}_{2} \mathrm{Cl}_{2}$ solution, the treatment with the dropwise addition of $\mathrm{BF}_{3} \cdot \mathrm{OEt}_{2}$ as catalyst induced $\mathrm{N}_{2}$ evolution. After complete conversion, the reaction mixture was neutralized with saturated $\mathrm{NaHCO}_{3}$ solution. The crude products were chromatographed on silica gel.

Mechanistically, it can be presumed [10] that the first step in the Schmidt reaction involves hemiacetal formation between the aromatic aldehyde and steroid azidoalcohol 2, which undergoes elimination to afford the benzyl carbocation 4. Intramolecular attack of the azide group on the carbocation furnishes intermediate $\mathbf{5}$, and subsequent proton elimination and $\mathrm{N}_{2}$ detachment give product 6 .

\subsection{Antiproliferative effect of the tested compounds}

The antiproliferative capacities of the newly synthesised D-ring modified estrogen analogues were tested against a panel of human gynaecological cancer cells containing cervical (HeLa, SiHa and C-33 A), ovarian (A2780) and breast (MCF-7, MDA- MB-231 and T47D) cancer cell lines (Table 1).

Our test compounds originating from $16 \alpha$-azido-3-methoxyestra$1,3,5(10)$ - trien-17 $\alpha$-ol (2), contain D-ring fused oxazoline ring with a substituted 2 '-phenyl moiety.

Based on the growth inhibition percentage and $\mathrm{IC}_{50}$ values calculated from the observed absorbance values it can be concluded that our test compounds possess moderate antiproliferative effects on all investigated cancer cell lines. The maximum value of cell growth inhibition at $30 \mu \mathrm{M}$ concentration of the tested molecules was around $70-80 \%$. Since $\mathrm{IC}_{50}$ values have been calculated if the growth inhibition value of the compound at $30 \mu \mathrm{M}$ concentration is higher than $75 \%$, IC $\mathrm{I}_{50}$ values have been determined only in the cases of compounds $6 \mathrm{e}, \mathrm{IC}_{50}(\mathrm{HeLa})=13.0 \mu \mathrm{M}$, and $6 \mathrm{~g}, \mathrm{IC}_{50}(\mathrm{MCF}-7)=16.5 \mu \mathrm{M}$. No substantial difference between the antiproliferative activities of the test compounds against cervical, ovarian or breast cancer cells has been demonstrated. However, based on the growth inhibition values inhibitory orders of the investigated molecules can be established on each cancer cell line. A comparison of these inhibitory orders reveals interesting structure-activity relationships in connection with the tested azido alcohol derivatives 6a-g. In general, formation of the D-ring fused oxazoline ring from the corresponding azido alcohol 2 slightly increased the cell growth inhibitory potential of the new compound. 6a in HeLa cells. Moreover, substitution of the phenyl ring with halogen atom resulted in compounds $(6 \mathbf{b}, \mathbf{6 c}$ and 6d) $6 \mathrm{~b}, \mathbf{6 c}$ and $\mathbf{6 d}$ with elevated antiproliferative activity against HeLa, HeLa and C-33A. From halogenated derivatives, the chlorine functional group generates the highest alteration in the growth inhibitory values on most of the investigated cancer cell lines. The strongest antiproliferative effect was observed in the case of compounds bearing 4"-nitrile, 4"-nitro or $4^{\prime \prime}$-methoxy-functional groups, because these molecules can be most frequently found among the three most effective compounds of the inhibitory orders (three times, six times and seven times, respectively). Compound $6 \mathrm{~g}$ with the $4^{\prime \prime}$-nitro function proved to be the most potent molecule against SiHa, A2780 and MCF-7 cells and the $4^{\prime \prime}$-methoxy derivative $6 \mathbf{e}$ inhibited cell proliferation with the highest activity on HeLa and MDA-MB-231 cells. The calculated $\mathrm{IC}_{50}$ value of $6 \mathrm{e}(12.9 \mu \mathrm{M})$ 
on HeLa cells is comparable to the $\mathrm{IC}_{50}$ value of cisplatin $(12.4 \mu \mathrm{M})$, our positive control compound, and a clinically used anticancer agent in the therapy of certain gynaecological malignancies. Both compounds, $6 \mathbf{e}$ and $6 \mathrm{~g}$, exhibited equal antiproliferative activity against T47D breast cancer cells.

In conclusion, the newly synthesized D-ring fused 2 '-phenyl oxazoline derivatives of $16 \alpha, 17 \alpha$ azido alcohol 2 demonstrated only moderate antiproliferative activity against the tested gynaecological cancer cell lines. However, the step-by-step consistent chemical modification of the D-ring fused 2 -phenylestrane backbone with a fused D-ring moiety illustrates its thorough impact on the pharmacological activity. Moreover, the $4^{\prime \prime}$-methoxy analogue $6 \mathrm{e}$ can be considered as a starting material in order to develop present-day still uncommon steroidal agents with higher anticancer potencies.

\section{Declaration of Competing Interest}

The authors declare that they have no known competing financial interests or personal relationships that could have appeared to influence the work reported in this paper.

\section{Acknowledgements}

The work of Anita Kiss was supported by a Ph.D. Fellowship of the Talentum of Richter Gedeon Plc. Financial support by National Research, Development and Innovation Office-NKFIH though Project [GINOP-2.3.2-15-2016-00038] is gratefully acknowledged. This research was supported by Hungarian Scientific Research Fund [OTKA SNN 124329].

\section{Appendix A. Supplementary data}

Supplementary data to this article can be found online at https://doi. org/10.1016/j.steroids.2021.108911.

\section{References}

[1] J.R. Hanson, Steroids: partial synthesis in medicinal chemistry, Nat. Prod. Rep. 27 (2010) 887-889.

[2] M. Ibrahim-Ouali, M. Santelli, Recent advences in thiosteroids chemistry, Steroids 71 (2006) 1025-1044.

[3] S.V. Stulov, A.Y. Misharin, Synthesis of steroids with nitrogen-containig substituents in ring D, Chem. Heterocyclic Com. 48 (2013) 1431-1472.

[4] É. Frank, G. Schneider, Synthesis of sex hormone-derived modified steroids possessing antiproliferative activity, J. Steroid Biochem. Mol. Biol. 137 (2013) 301-315.

[5] J. Wölfling, E. Mernyák, M. Sebők, G. Schneider, Synthesis of some steroidal oxazolines, Collect. Czech. Chem. Commun. 66 (12) (2001) 1831-1840.

[6] A. Hajnal, J. Wölfling, G. Schneider, novel preparation of dihydrooxazines condensed to ring D of the estrane skeleton, Synlett 7 (2002) 1077-1080.

[7] T.G. Gant, A.I. Meyers, The chemistry of 2-oxazolines (1985-present), Tetrahedron 50 (8) (1994) 2297-2360.

[8] U.T. Kim, S.K. Bhatia, J. Hajdu, Stereospeciphic synthesis of ether phospholipides. Preparation of 1-octadecyl-2-alakylaminodeoxglycerophospholipides, Tetrahedron Lett. 32 (1991) 6521-6524.

[9] K. Schwekendiek, F. Glorious, Efficient oxidative synthesis of 2-oxazolines, Synthesis (2006) 182996-183002.

[10] G.I. Koldobskii, V.A. Ostrovskii, B.V. Gidaspov, Schmidt reaction with aldehydes and carboxylic acids, Russ. Chem. Rev. 47 (11) (1978) 1084-1094.

[11] B. Schönecker, K. Ponsold, 16,17-Azido- und 16,17-aminoalkohole des Östra-1,3,5 (10)-trien-3-methylethers, Tetrahedron 31 (8) (1975) 1113-1118.

[12] T. Mosmann, Rapid colorimetric assay for cellular growth and survival: Application to proliferation and cytotoxicity assays, J. Immunol. Methods 65 (1-2) (1983) 55-63.

[13] S. Bräse, K. Banert, Organic Azide; Syntheses and Applications, Wiley and Sons Ltd., Chichester, United Kingdom, 2010, p. 191.

[14] J.H. Boyer, J. Hammer, The acid-catalysed reaction of alkyl azides upon carbonyl compounds, J. Am. Chem. Soc. 77 (1955) 951-954.

[15] J.G. Badiang, J. Aubé, One-step conversion of aldehydes to oxazolines and 5,6dihydro-4H-1,3-oxazines using 1,2- and 1,3-azido alkohols, J. Org. Chem. 61 (1996) 2484-2487.

[16] Molnár J, Frank É, Minorics R, Kádár Z, Ocsovszki, Schönecker B, Wölfling J, Zupkó I. A click approach to novel D-ring-substituted $16 \alpha$-triazolylestrone derivatives and characterization of their antiproliferative properties. PLOS One 10: 2 Paper: e0118104, 27 p. 2015.

[17] J.L. Luche, Lanthanides in organic chemistry. 1. Selective 1,2-reduction of conjugated ketones, J. Am. Chem. Soc. 100 (1978) 2226-2227.

[18] M. Leclaire, P. Jean, Inversion of the stereoselectivity in the reduction of the carbonyl group in a precursor to forskolin by the $\mathrm{NaBH} 4 / \mathrm{CeCl} 3$ reagent, Bull. Chim. Soc. Fr 133 (1996) 801-803.

[19] E. Št'astná, I. Černý, V. Pozuar, H. Chodounská, Stereoselectivity of sodium borohydride reduction on saturated steroidal ketones utilizing conditions of Luche reduction, Steroids 75 (2010) 721-725. 